\title{
CARACTERIZAÇÃO FÍSICO-QUÍMICA DE MANTEIGAS DE FRUTOS AMAZÔNICOS ${ }^{1}$
}

\author{
Neuza Jorge ${ }^{2}$ \\ Marcelo Vieira de Oliveira \\ Débora Maria Moreno Luzia
}

\begin{abstract}
RESUMO
Este trabalho teve como objetivo caracterizar manteigas comerciais obtidas a partir dos frutos amazônicos bacuri (Platonia insignis), cacau (Theobroma cacao), cupuaçu (Theobroma grandiflorum), murumuru (Astrocaryum murumuru), tucumã (Astrocaryum aculeatum) e ucuuba (Virola surinamensis) quanto às propriedades físico-químicas e ao perfil de ácidos graxos. Para tanto, as manteigas foram analisadas de acordo com os métodos oficiais descritos pela American Oil Chemists' Society (AOCS) quanto aos teores de ácidos graxos livres, índice de acidez, dienos conjugados, índice de peróxidos, $p$-anisidina, valor totox, índices de saponificação, iodo e refração, ponto de fusão e matéria insaponificável. As manteigas apresentaram acidez acima do limite estabelecido pela regulamentação, evidenciando degradação hidrolítica, sobretudo nas de cupuaçu e ucuuba. Ao contrário para os índices de peróxidos, a formação de compostos primários foi abaixo do limite máximo permitido pela regulamentação. A manteiga de bacuri apresentou o maior valor totox, pelos mais altos índices de peróxidos e $p$-anisidina. Em geral, as manteigas apresentaram elevadas quantidades de ácidos graxos saturados. Maiores quantidades de ácido graxo monoinsaturado (oleico) foram encontradas nas manteigas de cupuaçu, bacuri e cacau.
\end{abstract}

Palavras-chave: Ácidos graxos. Propriedades físico-químicas. Manteigas vegetais.

\section{PHYSICOCHEMICAL CHARACTERIZATION OF AMAZONIAN FRUIT BUTTERS}

\begin{abstract}
This study aimed to characterize commercial butters obtained from Amazonian fruits bacuri (Platonia insignis), cocoa (Theobroma cacao), cupuaçu (Theobroma grandiflorum), murumuru (Astrocaryum murumuru), tucumã (Astrocaryum aculeatum) and ucuuba (Virola surinamensis) regarding the physicochemical properties and the fatty acid profile. For this purpose, the butters were analyzed according to the official methods described by the American Oil Chemists' Society (AOCS) regarding the levels of free fatty acids, acidity index, conjugated dienes, peroxide index, $p$-anisidine, totox value, saponification, iodine and refraction, melting point and unsaponifiable matter. The butters showed acidity above the limit established by regulation, showing hydrolytic degradation, especially in cupuaçu and ucuuba. In contrast to the peroxide

\footnotetext{
${ }^{1}$ Como citar este artigo:

JORGE, N.; OLIVEIRA, M. V.; LUZIA, D. M. M. Caracterização físico-química de manteigas de frutos amazônicos. ForScience, Formiga, v. 9, n. 2, e00979, jul./dez. 2021. DOI: 10.29069/forscience.2021v9n2.e979.
}

\footnotetext{
${ }^{2}$ Autor para correspondência: Neuza Jorge, e-mail: neuza.jorge@unesp.br
} 
values, the formation of primary compounds was below the maximum limit allowed by regulation. Bacuri butter had the highest totox value, due to the higher levels of peroxides and $p$-anisidine. In general, the butters showed high amounts of saturated fatty acids. Higher amounts of monounsaturated fatty acid (oleic) were found in cupuaçu, bacuri and cocoa butters.

Keywords: Fatty acids. Physicochemical properties. Vegetable butters.

\section{INTRODUÇÃO}

O estudo da biodiversidade da vegetação amazônica tem despertado interesse dos pesquisadores, visando o manejo adequado e o melhor aproveitamento das espécies frutíferas na alimentação humana (COSTA; JORGE, 2012; JORGE; LUZIA, 2012). Além disso, existe o interesse dos pesquisadores na identificação de novas fontes de matéria-prima de baixo custo, com disponibilidade de nutrientes e elevado potencial industrial (SILVA; JORGE, 2014; BECKER et al., 2018).

Na região amazônica, a parte consumida dos frutos é geralmente a polpa que também é utilizada no processamento, produzindo sucos naturais, doces em conservas, extratos e polpas congeladas, com isso resíduos agroindustriais são gerados. Esses são formados por cascas, sementes e bagaços, podendo ser considerados fontes alternativas de alimentos e/ou matériaprima por conterem compostos bioativos (SILVA; JORGE, 2014).

Consequentemente, muita atenção tem sido dada à utilização de resíduos, principalmente das sementes. Ao invés de serem descartadas ou subutilizadas em rações animais e/ou fertilizantes, as sementes poderiam ser utilizadas na fortificação de alimentos, diminuindo os problemas de saúde pública, como a deficiência de minerais e vitaminas e, ao mesmo tempo, minimizando os problemas ambientais (SILVA; JORGE, 2019). Estudos já têm reportado que determinadas sementes de frutos amazônicos contêm relevantes quantidades de óleos e gorduras, entretanto, maiores investigações sobre suas propriedades são necessárias para avaliar o seu potencial como fontes de matéria-prima de boa qualidade para o consumo humano (COSTA-SINGH; BITENCOURT; JORGE, 2012; SINGH, 2015).

$\mathrm{O}$ aumento no interesse pelas matérias graxas de origem vegetal e seus derivados também está relacionado à gradual tendência de substituição na dieta humana das gorduras de origem animal, inclusive em países tradicionais, pelo consumo destas gorduras. Além disso, o uso de óleos e gorduras vegetais para fins não alimentícios como, por exemplo, pelas indústrias química, farmacêutica e cosmética, também tem contribuído para o aumento no consumo destas matérias graxas (LUZIA, 2012). 
A American Oil Chemists' Society tem proposto um conjunto de normas com limites específicos dos parâmetros de qualidades de óleos vegetais, de modo a garantir a conformidade dos mesmos dentro de um certo padrão de qualidade, levando em conta se eles estarão aptos ou não para consumo (AOCS, 2009). Dentre os parâmetros mais comumente analisados, encontram-se os ácidos graxos livres, ácidos dienóicos conjugados, índices de peróxidos, $\rho$ anisidina, saponificação, refração, iodo, estabilidade oxidativa, dentre outros (IAL, 2008).

A fim de explorar e utilizar os recursos naturais presentes no país, em especial os frutos amazônicos, este trabalho tem como objetivo caracterizar as manteigas comerciais extraídas dos frutos: bacuri (Platonia insignis), cacau (Theobroma cacao), cupuaçu (Theobroma grandiflorum), murumuru (Astrocaryum murumuru), tucumã (Astrocaryum aculeatum) e ucuuba (Virola surinamensis) quanto às propriedades físico-químicas e ao perfil de ácidos graxos.

\section{MATERIAL E MÉTODOS}

\subsection{Manteigas vegetais}

As manteigas vegetais provenientes dos frutos bacuri (Platonia insignis Mart.), cupuaçu (Theobroma grandiflorum) e murumuru (Astrocaryum murumuru) foram obtidas junto a uma empresa situada na cidade de Ananindeua, na região metropolitana de Belém, no Estado do Pará. As manteigas vegetais provenientes das amêndoas dos frutos cacau (Theobroma cacao), tucumã (Astrocaryum tucumã Mart.) e ucuuba (Virola sebifera Aubl.) foram adquiridas por uma empresa fornecedora situada na cidade de Catanduva, no Estado de São Paulo.

Logo após a chegada, cada lote de manteiga, pesando entre 800-1000 g, foi homogeneizado em recipiente de plástico e imediatamente acondicionado em frasco de vidro âmbar, inertizado com nitrogênio gasoso e armazenado a $-18^{\circ} \mathrm{C}$ até o momento das análises.

\subsection{Métodos}

Os ácidos graxos livres foram obtidos em titulador potenciométrico (modelo 794, Metrohm Ltd., Herisau, Switzerland), segundo método Ca 5a-40 da AOCS (2009), expressos em \% de ácido oleico. O índice de acidez foi calculado multiplicando-se a quantidade de ácidos graxos livres por 1,99, expresso em $\mathrm{mg} \mathrm{KOH} / \mathrm{g}$.

Os ácidos dienóicos conjugados foram medidos em espectrofotômetro a $233 \mathrm{~nm}$ (Modelo UV-VIS mini 1240, Shimadzu, Chiyoda-ku, Japão), de acordo com o método Ti 1a64 proposto pela AOCS (2009), expressos em porcentagem. 
O índice de peróxidos foi determinado empregando titulador potenciométrico (modelo 793, Metrohm Ltd., Herisau, Switzerland), conforme método Cd 8b-90 da AOCS (2009), expresso em meq $\mathrm{O}_{2} / \mathrm{kg}$.

O índice de $\rho$-anisidina ( $\mathrm{I} \rho \mathrm{A})$ foi determinado em espectrofotômetro (Modelo UV-VIS mini 1240, Shimadzu, Chiyoda-ku, Tokyo, Japão) a 350 nm, conforme o método Cd 18-90 da AOCS (2009).

O valor total de oxidação (totox) foi calculado por meio da equação: totox $=2(\mathrm{IP})+$ (I $\rho$ A) (ABREU et al., 2010).

O índice de saponificação foi calculado segundo o método Cd 3a-94 (AOCS, 2009) a partir da composição de ácidos graxos. Os resultados foram expressos em mg de $\mathrm{KOH} / \mathrm{g}$.

O índice de iodo foi calculado conforme o método Cd 1c-85 proposto pela AOCS (2009), por meio da composição de ácidos graxos, com os resultados expressos em g $\mathrm{I}_{2} / 100 \mathrm{~g}$.

O índice de refração foi realizado de acordo com o método Cc 7-25 da AOCS (2009), utilizando refratômetro de Abbé (Modelo UH 4, MLW Prüfgeräte, Berlim, Alemanha) a $40{ }^{\circ} \mathrm{C}$.

O ponto de fusão foi determinado pelo método do tubo capilar, imerso em água sob agitação e aquecimento, de acordo com o método Cc 1-25 da AOCS (2009), expresso em ${ }^{\circ} \mathrm{C}$.

A matéria insaponificável (MI) foianalisada segundo o método $\mathrm{Ca} 6 \mathrm{~b}-53$ proposto pela AOCS (2009), expressa em porcentagem.

O perfil dos ácidos graxos foi determinado pelo método da AOCS (2009), em cromatógrafo gasoso, a partir das manteigas esterificadas conforme método descrito por Hartman e Lago (1973). Utilizou-se um cromatógrafo a gás (3900, Varian Inc), equipado com detector de ionização de chama, sistema de injeção split de 1:30 e amostrador automático. Os compostos foram separados em coluna capilar de sílica fundida CP-Sil 88 de $60 \mathrm{~m}$ de largura, com diâmetro interno de $0,25 \mathrm{~mm}$ e espessura de filme de $0,20 \mu \mathrm{m}$. A temperatura inicial da coluna foi de $90{ }^{\circ} \mathrm{C}$ (durante quatro minutos) e programada para alcançar $195{ }^{\circ} \mathrm{C}$ com incremento de $10{ }^{\circ} \mathrm{C} / \mathrm{min}$, sendo, então, mantida em isoterma por 16 minutos. A temperatura do injetor foi de $230{ }^{\circ} \mathrm{C}$ e a do detector de $250{ }^{\circ} \mathrm{C}$. O gás de arraste foi o hidrogênio. Os ácidos graxos foram identificados de acordo com os tempos de retenção e a quantificação foi feita por normalização da área (\%). Utilizou-se como padrão uma mistura composta de 37 ésteres metílicos de ácidos graxos (Supelco, Bellefonte, USA), de C4:0 a C24:1, com pureza entre 99,1 e $99,9 \%$.

\subsection{Análise estatística}


Os resultados obtidos das determinações analíticas foram submetidos à análise de variância e as diferenças entre as médias foram testadas a $5 \%$ de probabilidade pelo teste de Tukey, por meio do programa STATISCA, versão 7.0.

\section{RESULTADOS E DISCUSSÃO}

Os resultados das análises para a caracterização físico-química das manteigas obtidas de frutos amazônicos estão apresentadas na Tabela 1. A formação de ácidos graxos livres decorre da hidrólise parcial dos triacilgliceróis. Excetuando a manteiga de bacuri, as demais apresentaram teores de ácidos graxos livres superiores ao valor permitido pelo Codex Alimentarium Commission (2009), que admite o máximo de $2 \%$ de ácidos graxos livres, expressos em ácido oleico, para a maioria dos óleos e gorduras prensados a frio e não-refinados. As manteigas cupuaçu, ucuuba e murumuru apresentaram as maiores porcentagens de ácidos graxos livres, 7,56; 6,14 e 5,76 \%, respectivamente, enquanto a manteiga da amêndoa de cacau obteve menor quantidade, 2,99 \%. Esses resultados indicam que tais manteigas estão inadequadas para o consumo.

Santos, Alves e Ruíz-Méndez (2013), em pesquisa com óleos extraídos de palmeiras amazônicas encontraram valor de ácidos graxos livres para óleo extraído do mesocarpo de tucumã de 1,9\%. Singh (2015) encontrou valores de ácidos graxos livres de 0,84 e 0,81\% para óleos da polpa e da amêndoa de tucumã, respectivamente. Isso aponta para uma qualidade inferior das manteigas usadas nesse estudo.

O limite estabelecido para o índice de acidez regulamentado pelo Codex Alimentarium Commission (2009) para óleos e gorduras prensados a frio e não-refinados deve ter no máximo 4,0 mg de KOH/g. Segundo Farhoosh, Einafshar e Sharayei (2009), o índice de acidez reflete o grau de conservação do óleo que está diretamente relacionado com a natureza e qualidade da matéria-prima, com os processos de extração e refinação, e, principalmente, com as condições de armazenamento, pois a decomposição dos glicerídeos é acelerada por ação enzimática, aquecimento e luminosidade.

Os ácidos dienóicos conjugados ou dienos conjugados são formados pela mudança de posição da dupla ligação nos ácidos graxos poli-insaturados que sofrem peroxidação formando os hidroperóxidos conjugados (SHAHIDI, 2005). A maior formação de dienos na matéria graxa indica formação de compostos primários de oxidação lipídica (McCLEMENTS; DECKER, 2007). 
As manteigas de bacuri e ucuuba apresentaram teores de dienos conjugados significativamente maiores quando comparadas às demais, 2,60 e $2,74 \%$, respectivamente. Tais resultados evidenciam que o processo de oxidação está mais avançado nestas matérias graxas, o que significa que na extração ou em alguma etapa do processamento podem ter sido mais expostas à ação da luz, temperatura e oxigênio, fatores esses que aceleram o processo de oxidação.

Tabela 1 - Caracterização físico-química das manteigas extraídas de frutos amazônicos

\begin{tabular}{|c|c|c|c|c|c|c|}
\hline Análises & Bacuri & Cacau & Cupuaçu & Murumuru & Tucumã & Ucuuba \\
\hline AGL (\% oleico) & nd & $2,99 \pm 0,04^{\mathrm{e}}$ & $7,56 \pm 0,02^{\mathrm{a}}$ & $5,76 \pm 0,03^{c}$ & $4,65 \pm 0,02^{\mathrm{d}}$ & $6,14 \pm 0,00^{b}$ \\
\hline IA (mg KOH/g) & nd & $5,95 \pm 0,04^{\mathrm{e}}$ & $15,04 \pm 0,02^{\mathrm{a}}$ & $11,46 \pm 0,03^{c}$ & $9,26 \pm 0,02^{\mathrm{d}}$ & $12,21 \pm 0,00^{\mathrm{b}}$ \\
\hline $\mathrm{DC}(\%)$ & $2,60 \pm 0,00^{\mathrm{b}}$ & $0,18 \pm 0,00^{\mathrm{f}}$ & $0,44 \pm 0,00^{\mathrm{c}}$ & $0,23 \pm 0,00^{\mathrm{e}}$ & $0,26 \pm 0,00^{\mathrm{d}}$ & $2,76 \pm 0,01^{\mathrm{a}}$ \\
\hline IP (meq/kg) & $13,31 \pm 0,06^{\mathrm{a}}$ & $0,21 \pm 0,01^{\mathrm{f}}$ & $11,63 \pm 0,11^{\mathrm{b}}$ & $2,83 \pm 0,12^{\mathrm{c}}$ & $1,21 \pm 0,00^{\mathrm{e}}$ & $2,20 \pm 0,14^{\mathrm{d}}$ \\
\hline $\mathbf{I} \rho \mathbf{A}$ & $9,85 \pm 0,00^{\mathrm{b}}$ & $1,81 \pm 0,11^{\mathrm{e}}$ & $0,59 \pm 0,01^{\mathrm{f}}$ & $4,28 \pm 0,05^{\mathrm{d}}$ & $11,12 \pm 0,11^{\mathrm{a}}$ & $6,63 \pm 0,01^{\mathrm{c}}$ \\
\hline VT & $36,47 \pm 0,13^{a}$ & $2,23 \pm 0,14^{\mathrm{f}}$ & $23,85 \pm 0,25^{\mathrm{b}}$ & $9,94 \pm 0,05^{\mathrm{e}}$ & $13,54 \pm 0,11^{\mathrm{c}}$ & $11,03 \pm 0,27^{\mathrm{d}}$ \\
\hline IS (mg KOH/g) & $199,93 \pm 0,03^{\mathrm{d}}$ & $195,86 \pm 0,05^{\mathrm{e}}$ & $191,68 \pm 0,01^{\mathrm{f}}$ & $234,74 \pm 0,03^{b}$ & $237,93 \pm 0,01^{\mathrm{a}}$ & $234,34 \pm 0,01^{\mathrm{c}}$ \\
\hline II (g I/2/100 g) & $38,00 \pm 0,02^{\mathrm{b}}$ & $29,79 \pm 0,06^{\mathrm{c}}$ & $61,02 \pm 0,09^{\mathrm{a}}$ & $19,26 \pm 0,33^{\mathrm{d}}$ & $15,73 \pm 0,03^{\mathrm{e}}$ & $4,37 \pm 0,03^{\mathrm{f}}$ \\
\hline $\operatorname{IR}\left(40^{\circ} \mathrm{C}\right)$ & $1,4590 \pm 0,0000^{\mathrm{a}}$ & $1,4580 \pm 0,0000^{\mathrm{ab}}$ & $1,4570 \pm 0,0000^{\mathrm{ab}}$ & $1,4525 \pm 0,0000^{\mathrm{b}}$ & $1,4516 \pm 0,0000^{\mathrm{b}}$ & $1,4520 \pm 0,0000^{\mathrm{b}}$ \\
\hline PF $\left({ }^{\circ} \mathrm{C}\right)$ & $34,00 \pm 0,00^{\mathrm{b}}$ & $22,00 \pm 0,00^{\mathrm{e}}$ & $29,50 \pm 0,00^{\mathrm{d}}$ & $32,00 \pm 0,00^{\mathrm{c}}$ & $29,80 \pm 0,00^{\mathrm{d}}$ & $42,00 \pm 0,00^{\mathrm{a}}$ \\
\hline MI (\%) & $5,94 \pm 0,07^{\mathrm{a}}$ & $2,07 \pm 0,10^{\mathrm{c}}$ & $2,25 \pm 0,02^{\mathrm{c}}$ & $1,63 \pm 0,30^{\mathrm{d}}$ & $1,40 \pm 0,07^{\mathrm{d}}$ & $5,36 \pm 0,05^{\mathrm{b}}$ \\
\hline
\end{tabular}

a, b... (linha): médias \pm desvios padrões seguidas de mesma letra minúscula não diferem pelo teste de Tukey $(\mathrm{p}>0,05)$.

ácidos graxos livres (AGL), índice de acidez (IA), dienos conjugados (DC), índice de peróxidos (IP), índice de $p$-anisidina( I $\rho$ A), valor totox (VT), índice de saponificação (IS), índice de iodo (II); índice de refração (IR), ponto de fusão (PF), matéria insaponificável (MS).

nd: não detectado. 
O índice de peróxidos é usado como parâmetro de qualidade para avaliar a formação de produtos primários da oxidação em óleos e gorduras. O Codex Alimentarium Commission (2009) estabelece para índice de peróxidos para óleos virgens ou prensados a frio o valor máximo de $15 \mathrm{meq} / \mathrm{kg}$. Os valores de peróxidos das manteigas analisadas estão abaixo desse limite.

Singh (2015), estudando óleos de frutos amazônicos, encontrou elevado índice de peróxidos para o óleo da polpa de bacabá $(59,31 \mathrm{meq} / \mathrm{kg})$, o que, segundo a autora, pode ser explicado pelo fato desses frutos terem apenas uma película envolvendo a polpa, que, dessa forma, fica mais exposta à ação da luz, temperatura e oxigênio, fatores esses que aceleram o processo de oxidação e a formação de peróxidos. Altos valores de índice de peróxidos indicam que, de alguma forma, a matéria graxa foi exposta ao processo oxidativo durante o preparo da matéria-prima, extração ou armazenamento da matéria graxa. Por outro lado, um baixo nível de peróxidos não indica garantia de estabilidade oxidativa, uma vez que estes compostos podem ser degradados.

De acordo com resultados encontrados, as manteigas de cupuaçu e ucuuba apresentaram maior degradação hidrolítica, evidenciada pelos elevados teores de ácidos graxos livres e acidez; enquanto que as manteigas de bacuri e cupuaçu apresentaram maior degradação oxidativa, representada pelo valor totox, em relação às demais manteigas avaliadas nesse estudo.

O índice de $p$-anisidinapode ser utilizado na avaliação da deterioração do óleo e/ou gordura durante a termoxidação, uma vez que o valor tende a aumentar com o tempo de aquecimento, de forma linear. Além disso, é um teste que se correlaciona com as determinações de ácidos dienóicos conjugados e compostos polares totais. Uma matéria graxaconsiderada de boa qualidade deve apresentar um IpA inferior a 10 (GUILLÉN; CABO, 2002). O índice de $\rho$ anisidina mede os produtos secundários formados a partir da decomposição de peróxidos presentes no óleo oxidado, considerados compostos intermediários e transitórios (SHAHIDI; WANASUNDARA, 2008). Com exceção da manteiga de tucumã, que apresentou um valor de I $\rho$ A de 11,12; as demais manteigas apresentaram valores inferiores a 10, com destaque para a de cupuaçu com menor índice $(0,60)$.

Valor total de oxidação é a combinação entre o valor de índices de peróxidos e $\rho$ anisidina. O nível de peróxidos representa o potencial de degradação da qualidade sensorial e $\rho$-anisidina, mostra o estado de deterioração efetivo. Segundo Berset e Culivier (1996), uma matéria graxa bem conservada apresenta valor totoxinferior a 10. Apenas as manteigas de cacau e murumuru apresentaram valores Totox abaixo desse limite aceitável, 2,23 e 9,94, 
respectivamente. A manteiga de cacau apresentou o menor valor totox, logicamente pelos baixos índices de peróxidos $(0,21 \mathrm{meq} / \mathrm{kg})$ e $p$-anisidina $(1,81)$.

O índice de saponificação é definido como a quantidade de hidróxido de potássio $(\mathrm{KOH})$, necessários para saponificar os ácidos graxos, resultantes da hidrólise de um grama da amostra.

Conforme Ribeiro e Seravalli (2007), a reação de saponificação pode estabelecer o grau de deterioração e a estabilidade, verificar se as propriedades dos óleos estão de acordo com as especificações e identificar possíveis fraudes e adulterações.

Dentre as manteigas estudadas, o menor índice de saponificação ocorreu para a manteiga de cupuaçu (191,68 mg KOH/g). O índice de saponificação revela o peso molecular médio dos ácidos graxos esterificados com o glicerol na molécula de triacilglicerol, ou seja, um índice de saponificação baixo sugere ácidos de pesos moleculares elevados. Por outro lado, o maior índice de saponificação foi atribuído à manteiga de tucumã (237,93 mg KOH/g), valor próximo ao encontrado por Singh (2015), para o óleo de amêndoas de pupunha (234, $87 \mathrm{mg} \mathrm{KOH} / \mathrm{g}$ ).

O índice de iodo é um teste que mede o grau de insaturação de um óleo ou gordura. Os valores encontrados para as manteigas neste estudo variaram de 4,37 a 61,02 $\mathrm{I}_{2} / 100 \mathrm{~g}$. A manteiga de cupuaçu apresentou também elevado valor de índice de refração a $40{ }^{\circ} \mathrm{C}(1,4570)$ e maior teor de insaturados $(55,45 \%)$, demonstrando, dentre as manteigas estudadas, a que apresenta maior insaturação, ou seja, uma maior predisposição à oxidação lipídica. De acordo com Van De Mark e Sandefur (2005), as manteigas estudadas são classificadas como nãosecativas (II < $115 \mathrm{I}_{2} / 100 \mathrm{~g}$ ), ou seja, apresentam maior quantidade de ácidos graxos saturados e não são capazes de polimerizar ao formar um filme elástico pela absorção de oxigênio do ar.

Em estudo com óleos extraídos da polpa de frutos amazônicos, Singh (2015) encontrouos valores de índice de iodo 60,46 e 62,12 g I $/ 100 \mathrm{~g}$ para pupunha e tucumã, respectivamente. Estes resultados se aproximam ao encontrado para a manteiga de cupuaçu $(61,02 \mathrm{~g} \mathrm{I} / 100 \mathrm{~g})$

O índice de refração tem relação inversa com o grau de saturação das moléculas. O índice de refração é um parâmetro que pode ser influenciado pelo comprimento de cadeia e grau de insaturação dos ácidos graxos. Também pode ser afetado por alguns fatores como teor de ácidos graxos livres, oxidação e tratamento térmico (GUNSTONE, 2011).

Os valores de índice de refração para as manteigas estudadas variaram de 1,4516 a 1,4590. As manteigas de tucumã e ucuuba apresentaram os menores índices de refração, 1,4516 e 1,4520, respectivamente. Isso indica que possuem menor quantidade de insaturações, tendo uma maior estabilidade por conta disso. Esses valores são próximos aos encontrados por 
Coimbra e Jorge (2011) para óleos extraídos de polpas de guariroba e macaúba, 1,4535 e 1,4556, respectivamente. Da mesma forma, as manteigas de bacuri, cacau e cupuaçu apresentaram maiores índices de refração $(1,4590,1,4580$ e 1,4570, respectivamente), sugerindo que essas matérias graxas sejam menos estáveis.

De acordo com a Tabela 1 pode-se observar que, à exceção da manteiga de cacau, as demais apresentaram ponto de fusão elevado, com destaque para ucuuba $\left(42^{\circ} \mathrm{C}\right)$. $\mathrm{O}$ ponto de fusão está diretamente relacionado com o número de saturações dos ácidos graxos (95,68 \%), conferindo-lhe maior ponto de fusão. Essa característica é observada como condição favorável para a utilização de manteigas vegetais, como ocorre para a manteiga de cupuaçu, como substituto parcial para a manteiga de cacau, impedindo que produtos como barras de chocolate amoleçam rapidamente em ambientes com temperaturas mais elevadas, como ocorre costumeiramente em algumas regiões do Brasil.

A matéria insaponificável corresponde aos compostos presentes nas matérias graxas que após saponificação com álcalis são insolúveis em solução aquosa, incluindo substâncias naturalmente presentes, como esteróis, tocoferóis, carotenóides. Souza et al. (2007), em estudo com os óleos das amêndoas de pistache e macadâmia, encontraram teores de matéria insaponificável no intervalo de 0,45-0,64 \% e 1,17-1,39\%, respectivamente. Com exceção da manteiga de tucumã, as demais apresentaram porcentagens de matéria insaponificável superiores aos valores citados no Codex Alimentarium Commission (2009) para óleos vegetais como milho $(\leq 2,8 \%)$, girassol $(\leq 1,5 \%)$ e soja $(\leq 1,5 \%)$. O elevado valor de matéria insaponificável pode ser interessante para a indústria cosmética ou alimentícia, que pode incorporar esteróis, tocoferóis e carotenoides dessas manteigas na formulação de seus produtos.

De acordo com a Tabela 2, dos 11 ácidos graxos identificados, apenas quatro ácidos estiveram presentes em todas as manteigas, a saber: palmítico, esteárico, oleico e linoleico. Em grande parte das manteigas predominaram os ácidos graxos saturados, com destaque para palmítico, mirístico, láurico e esteárico. A manteiga de ucuuba se destaca com 95,68\% de saturados, com ênfase para o ácido mirístico (71,47\%). Por outro lado, apenas a manteiga de cupuaçu apresentou como majoritário, o ácido oleico (46,63\%). O ácido palmítico, que normalmente é o ácido graxo saturado mais abundante nos lipídios vegetais, ocorreu em percentuais consideráveis para todas as manteigas, com destaque para as manteigas de bacuri $(46,74 \%)$ e cacau $(36,03 \%)$. Já o ácido láurico foi predominante nas manteigas de murumuru $(44,99 \%)$ e tucumã $(49,95 \%)$. De acordo com Singh (2015), nos produtos em que a presença da gordura sólida é indispensável para a manutenção da textura e da consistência, a substituição da gordura vegetal hidrogenada, com elevados teores de ácidos graxostrans, pela gordura 
saturada, com elevados teores de ácido láurico, parece ser uma alternativa interessante, uma vez que este tipo de gordura resulta em um perfil lipídico sanguíneo mais favorável do que uma gordura sólida rica em ácidos graxos trans.

O ácido graxo esteárico foi majoritário na manteiga de cacau $(32,03 \%)$ com um teor muito próximo de ácido oleico $(29,96 \%)$. Essa condição ocorreu também para o cupuaçu, com a diferença de que neste, o ácido oleico teve um maior teor que o ácido esteárico, 46,63 \% e 33,69 \%, respectivamente. Embora se saiba que o consumo de gorduras saturadas aumenta o nível plasmático de colesterol (BAUM et al., 2012), o consumo regular de manteiga de cacau e chocolate vem negando esse aumento. Isso se deve, provavelmente, às concentrações relativamente altas de ácido esteárico, que tem mostrado um efeito neutro sobre o metabolismo do colesterol, e ao ácido oleico, que, apesar de não ser considerado um ácido graxo essencial, exerce um efeito neutro sobre a colesterolemia, aumentando a fração HDLc, reduzindo o colesterol total, o nível da fração LDLc e a incidência de doenças cardíacas. Além disso, auxilia na prevenção do câncer, exerce ação antitrombótica e inibe a agregação plaquetária. Estes ácidos graxos estão presentes tanto em espécies vegetais como animais (MAHAN; ESCOTTSTUMP, 2010).

Os ácidos caprílico e cáprico foram detectados nas manteigas de murumuru, tucumã e ucuuba, em teores que variaram de 0,22 a 1,60 \%. Ainda que em pequena quantidade, somente a manteiga de tucumã apresentou o ácido capróico.

O ácido oleico, apesar de estar presente em todas as manteigas analisadas (3,62 a 46,63 $\%$ ), foi o único ácido graxo monoinsaturado detectado, sobressaindo nas manteigas de cupuaçu (46,63\%) e bacuri (37,62 \%). De acordo com Lopez-Huertas (2010), a presença de ácido oleico na dieta humana diminui os níveis da fração LDLc no sangue, a pressão sanguínea e a incidência de doenças cardíacas.

Os poli-insaturados totais variaram de 0,69 a 8,82 \%, incluindo linoleico e $\alpha$-linolênico, ácidos graxos essenciais à espécie humana. O ácido linoleico apresentou em maior quantidadena manteiga de murumuru $(6,14$ \%). Com relação ao ácido $\alpha$-linolênico, a manteiga de cupuaçu foi a que apresentou maior percentagem $(6,67 \%)$.

Em geral, as manteigas apresentaram elevadas quantidades de ácidos graxos saturados. Maiores quantidades de ácido graxo monoinsaturado (oleico) foram encontradas nas manteigas de cupuaçu, bacuri e cacau (Tabela 2).

Comparando-se a relação entre o total de ácidos graxos saturados e insaturados das manteigas analisadas neste trabalho com os resultados obtidos por Singh (2015) para óleos de 
amêndoas de frutos amazônicos, verificou-se que a manteiga de murumuru $(1 / 0,19)$ aproximou-se mais ao óleo de inajá $(1 / 0,21)$.

Tabela 2 - Composição em ácidos graxos das manteigas extraídas de frutos amazônicos

\begin{tabular}{|c|c|c|c|c|c|c|}
\hline Ácidos Graxos (\%) & Bacuri & Cacau & Cupuaçu & Murumuru & Tucumã & Ucuuba \\
\hline Saturados & $59,35 \pm 0,02^{\mathrm{e}}$ & $68,06 \pm 0,02^{\mathrm{d}}$ & $44,64 \pm 0,02^{\mathrm{f}}$ & $84,09 \pm 0,02^{\mathrm{c}}$ & $85,39 \pm 0,02^{b}$ & $95,68 \pm 0,02^{\mathrm{a}}$ \\
\hline Capróico (C6:0) & nd & nd & nd & nd & $0,18 \pm 0,01$ & nd \\
\hline Caprílico (C8:0) & $\operatorname{tr}$ & nd & nd & $1,37 \pm 0,02^{b}$ & $1,59 \pm 0,01^{\mathrm{a}}$ & $0,22 \pm 0,01^{\mathrm{c}}$ \\
\hline Cáprico (C10:0) & $\operatorname{tr}$ & nd & nd & $1,24 \pm 0,01^{b}$ & $1,60 \pm 0,00^{\mathrm{a}}$ & $0,73 \pm 0,03^{\mathrm{c}}$ \\
\hline Láurico (C12:0) & $1,27 \pm 0,02^{\mathrm{d}}$ & nd & nd & $44,99 \pm 0,38^{b}$ & $49,95 \pm 0,14^{\mathrm{a}}$ & $18,37 \pm 0,10^{c}$ \\
\hline Mirístico (C14:0) & $0,90 \pm 0,01^{\mathrm{d}}$ & nd & nd & $26,11 \pm 0,00^{\mathrm{b}}$ & $23,35 \pm 0,06^{\mathrm{c}}$ & $71,47 \pm 0,05^{\mathrm{a}}$ \\
\hline Palmítico (C16:0) & $46,74 \pm 0,03^{\mathrm{a}}$ & $36,03 \pm 0,01^{b}$ & $11,15 \pm 0,08^{c}$ & $6,96 \pm 0,08^{d}$ & $5,85 \pm 0,04^{\mathrm{e}}$ & $4,04 \pm 0,02^{\mathrm{f}}$ \\
\hline Esteárico (C18:0) & $7,16 \pm 0,00^{\mathrm{c}}$ & $32,03 \pm 0,04^{b}$ & $33,69 \pm 0,11^{\mathrm{a}}$ & $3,20 \pm 0,06^{\mathrm{d}}$ & $2,78 \pm 0,01^{\mathrm{e}}$ & $0,66 \pm 0,02^{\mathrm{f}}$ \\
\hline Araquídico (C20:0) & $3,28 \pm 0,01^{\mathrm{a}}$ & nd & nd & $0,13 \pm 0,0^{\mathrm{c}}$ & $\operatorname{tr}$ & $0,18 \pm 0,03^{b}$ \\
\hline Monoinsaturados & $37,62 \pm 0,01^{b}$ & $29,96 \pm 0,03^{c}$ & $46,63 \pm 0,09^{\mathrm{a}}$ & $9,77 \pm 0,17^{\mathrm{e}}$ & $11,06 \pm 0,05^{\mathrm{d}}$ & $3,62 \pm 0,02^{f}$ \\
\hline Oleico (C18:1) & $37,62 \pm 0,01^{\mathrm{b}}$ & $29,96 \pm 0,03^{c}$ & $46,63 \pm 0,09^{\mathrm{a}}$ & $9,77 \pm 0,17^{\mathrm{e}}$ & $11,06 \pm 0,05^{\mathrm{d}}$ & $3,62 \pm 0,02^{f}$ \\
\hline Poli-insaturados & $3,02 \pm 0,02^{\mathrm{d}}$ & $2,00 \pm 0,02^{\mathrm{e}}$ & $8,82 \pm 0,02^{\mathrm{a}}$ & $6,14 \pm 0,11^{b}$ & $3,53 \pm 0,01^{\mathrm{c}}$ & $0,69 \pm 0,01^{\mathrm{f}}$ \\
\hline Linoleico (C18:2) & $2,58 \pm 0,08^{\mathrm{c}}$ & $1,34 \pm 0,01^{\mathrm{e}}$ & $2,15 \pm 0,00^{\mathrm{d}}$ & $6,14 \pm 0,11^{\mathrm{a}}$ & $3,53 \pm 0,01^{b}$ & $0,69 \pm 0,01^{\mathrm{f}}$ \\
\hline$\alpha$-Linolênico (C18:3) & $0,44 \pm 0,04^{\mathrm{c}}$ & $0,66 \pm 0,01^{\mathrm{b}}$ & $6,67 \pm 0,06^{\mathrm{a}}$ & $\operatorname{tr}$ & $\operatorname{tr}$ & $\operatorname{tr}$ \\
\hline Sat/Insat* & $1 / 0,68$ & $1 / 0,47$ & $1 / 1,24$ & $1 / 0,19$ & $1 / 0,17$ & $1 / 0,05$ \\
\hline
\end{tabular}

a, b... (linha): médias \pm desvios padrões seguidas de mesma letra minúscula não diferem pelo teste de Tukey $(\mathrm{p}>0,05)$.

nd: não detectado; tr: porcentagem menor que $0,1 \%$.

*Relação entre o total de ácidos graxos saturados e insaturados.

Acredita-se que o consumo excessivo de óleos contendo ácidos graxos saturados com cadeias de 12 a 16 carbonos aumente a concentração de lipoproteínas de baixa densidade (LDL) no sangue e eleve o risco de doenças cardiovasculares (SCHAEFER, 1997). Os ácidos graxos 
monoinsaturados e poli-insaturados tendem a apresentar uma relação inversa com a incidência de doenças coronarianas (BINKOSKI et al., 2005).

\section{CONCLUSÃO}

As manteigas apresentaram acidez acima do limite estabelecido pela regulamentação, evidenciando degradação hidrolítica, sobretudo nas de cupuaçu e ucuuba. Ao contrário para os índices de peróxidos, a formação de compostos primários foi abaixo do limite máximo permitido pela regulamentação, indicando que as manteigas foram processadas seguindo as boas práticas de fabricação. A manteiga de bacuri apresentou o maior valor totox, logicamente pelos mais altos índices de peróxidos e $p$-anisidina.

Nas manteigas avaliadas houve predominância dos ácidos graxos saturados, com destaque para palmítico, mirístico, láurico e esteárico. $\mathrm{O}$ ácido láurico foi predominante nas manteigas demurumuru e tucumã. A manteiga de ucuuba se destacou com 95,68 \% de saturados, com ênfase para o ácido mirístico. O ácido palmítico ocorreu em percentuais consideráveis para todas as manteigas, com destaque para as manteigas de bacuri e cacau.O ácido graxo esteárico foi majoritário nas manteigasde cupuaçu e cacau. Por outro lado, maiores quantidades de ácidooleico foram encontradas nas manteigas de cupuaçu, bacuri e cacau.

Conclui-se que as manteigas podem ser utilizadas tanto nas indústrias alimentícia, cosmética e farmacêutica, por oferecerem maior consistência e estabilidade oxidativa aos produtos.

\section{AGRADECIMENTOS}

À Coordenação de Aperfeiçoamento de Pessoal de Nível Superior (CAPES), pelo auxílio financeiro e bolsa de doutorado; à Universidade Estadual Paulista "Júlio de Mesquita Filho" (UNESP); ao Instituto Federal de Educação, Ciência e Tecnologia do Maranhão (IFMA) e ao Conselho Nacional de Desenvolvimento Científico e Tecnológico (CNPq), pela bolsa de produtividade em pesquisa.

\section{REFERÊNCIAS}

ABREU, D. A. P. de; LOSADA, P. P.; MAROTO, J.; CRUZ, J. M. Evaluation of the effectiveness of a antioxidants (from barley husks) that retard lipid damage in frozen atlantic 
salmon (Salmo salar L.). Food Research International, Barking, v. 43, n. 5, p.1277-1282, 2010 .

AMERICAN OIL CHEMITS' SOCIETY. Official and Tentative Methods of the American Oil Chemists' Society: including additions and revisions. 6. ed. Champaing: AOCS Press, 2009.

BAUM, S. J. et al. Fatty acids in cardiovascular health and disease: a comprehensive update. Journal of Clinical Lipidology, Atlanta, v. 6, n. 3, p. 216-234, 2012.

BECKER, M. M. et al. Mineral and bromatological assessment and determination of the antioxidant capacity and bioactive compounds in native amazon fruits. Brazilian Journal of Food Technology, Campinas, v. 21, e2018022, 2018.

BERSET, C.; CUVELIER, M. E. Methods of estimating the degree of lipid oxidation and of measuring antioxidizing power. Sciences des Aliments, Dijon, v. 16, n. 3, p. 219-245, 1996.

BINKOSKI, A. E. et al. Balance of unsaturated fatty acids is important to a cholesterol lowering diet: comparison of mid-oleic sunflower oil and olive oil on cardiovascular disease risk factors. Journal of the American Dietetic Association, Chicago, v. 105, n. 7, p. 10801086, 2005.

CODEX ALIMENTARIUS COMISSION.Codex-Stan 210: Codex Standard for named vegetable oils, Codex Alimetarius Comission, Rome; 2009.

COIMBRA, M. C.; JORGE, N. Characterization of the pulp and kernel oils from Syagrus oleracea, Syagrus romanzoffiana and Acrocomia aculeate. Journal of Food Science, Chicago, v. 76, n. 8, p. 1156-1161, 2011.

COSTA, T.; JORGE, N. Characterization and fatty acids profile of the oils from amazon nuts and walnuts: characterization and fatty acids profile of the oil seeds. Nutrition \& Food Science, London, v. 42, n. 4, p. 279-287, 2012.

COSTA-SINGH, T.; BITENCOURT, T. B.; JORGE, N. Caracterização e compostos bioativos do óleo da castanha-de-cutia (Couepia edulis). Revista do Instituto Adolfo Lutz, São Paulo, v. 71, n. 1, p. 61-8, 2012.

FARHOOSH, R.; EINAFSHAR, S.; SHARAYEI, P. The effect of commercial refining steps on the rancidity measures of soybean and canola oils. Food Chemistry, London, v. 115, n. 3, p. 933-938, 2009.

GUILLÉN, M. D.; CABO, N. Fourier transform infrared spectra data versus peroxide and anisidine values to determine oxidative stability of edible oils. Food Chemistry, London, v. 77, n. 4, p. 503-510, 2002.

GUNSTONE, F. D. Vegetable oils in food technology: composition, properties and uses. 2 ed. Oxford: Wiley-Blackwell, 2011, 376 p.

HARTMAN, L.; LAGO, R. C. A. Rapid preparation of fatty acid methyl esters from lipids. Laboratory Practice, London, v. 22, p. 475-476, 1973. 
INSTITUTO ADOLFO LUTZ (IAL). Normas analíticas, métodos químicos e físicos para análises de alimentos. 4. ed. São Paulo: Instituto Adolfo Lutz, 2008. 533 p.

JORGE, N.; LUZIA, D. M. M. Caracterização do óleo das sementes de Pachira aquatica Aublet para aproveitamento alimentar. Acta Amazonica, Manaus, v. 42, n. 1, p. 149-156, 2012.

LOPEZ-HUERTAS, E. Health effects of oleic acid and long chain omega-3 fatty acids (EPA and DHA) enriched milks. A review of intervention studies. Pharmacological Research, London, v. 61, n. 3, p. 200-207, 2010.

LUZIA, D. M. M. Propriedades funcionais de óleos extraídos de sementes de frutos do cerrado brasileiro. 2012. 234 p. Tese (Doutorado em Engenharia e Ciência de Alimentos) Universidade Estadual Paulista, São José do Rio Preto, 2012.

MAHAN, L.K.; ESCOTT-STUMP, S. Krause: alimentos, nutrição e dietoterapia. 12 ed. São Paulo: Roca, 2010. 1351 p.

McCLEMENTS, D. J.; DECKER, E. A. Lipids. In: DAMODARAN, S.; PARKIN, K. L.; FENNEMA, O. R. Food chemistry. 4. ed. USA: CRC Press, 2007. p. 155-216.

RIBEIRO, E. P.; SERAVALLI, E. A. G. Química de alimentos. 2 ed. São Paulo: Edgar Blucher Ltda, 2007. 196 p.

SANTOS, M. F. G.; ALVES, R. E.; RUÍZ-MÉNDEZ, M. V. Minor components in oils obtained from Amazonian palm fruits. Grasas y Aceites, Sevilha, v. 64, n. 5, p. 531-536, 2013.

SCHAEFER, E. J. Effects of dietary fatty acids on lipoproteins and cardiovascular disease risk: summary. American Journal of Clinical Nutrition, New York, v. 65, p. 1655S-1656S, 1997.

SHAHIDI, F. Bailey's industrial oil \& fats products. 6 ed. New York: John Wiley \& Son 2005. $3616 \mathrm{p}$.

SHAHIDI, F.; WANASUNDARA, U. N. Methods for measuringoxidativerancidity in fatsandoils. In: AKOH, C. C.; MIN, D. B. Food lipids: chemistry, nutrition and biotechnology. 3 ed. USA: CRC Press, 2008. p. 387-407.

SILVA, A. C.; JORGE, N. Bioactive compounds of the lipid fractions of agro-industrial waste. Food Research International, Barking, v.66, p. 493-500, 2014.

SILVA, A. C.; JORGE, N. Bioactive properties and antioxidant capacity of oils extracted from citrus fruit seeds. Acta Alimentaria, Budapest, v. 48, n. 2, p. 196-203, 2019.

SINGH, T. C. Avaliação dos parâmetros físico-químicos e estabilidade de compostos bioativos em óleos de polpa e amêndoa de frutos amazônicos. 2015. 158 p. Tese (Doutorado em Engenharia e Ciência de Alimentos) - Universidade Estadual Paulista, São José do Rio Preto, 2015. 
SOUZA, D. F. S. et al. Estabilidade oxidativa dos óleos de macadâmia e de pistache. Boletim do Centro de Pesquisa e Processamento de Alimentos, Curitiba, v. 25, n. 1, p. 141-156, 2007.

VAN DE MARK, M. R.; SANDEFUR, K. Vegetable oils in paint and coatings. In: ERHAN, S. Z. Industrial uses of vegetable oils. Champaign: AOCS Press, 2005. p. 143-162.

\section{DADOS DOS AUTORES:}

Nome: Marcelo Vieira de Oliveira

E-mail: marcelovieira@ifma.edu.br

Curriculum Lattes: http://lattes.cnpq.br/5900242911044711

Graduação em Licenciatura em Ciências Agrícolas pela Universidade Federal Rural do Rio de Janeiro, especialização em Solos e Meio Ambiente pela UFLA-MG, especialização em Educação de Jovens e Adultos pelo CEFET-MA e mestrado em Ciência e Tecnologia de Alimentos pela UFRRJ. Atualmente é professor/ensino básico, técnico e tecnológico do Instituto Federal do Maranhão. Tem experiência em Ciências Agrárias, com ênfase em Agricultura e Tecnologia de Alimentos.

Nome: Débora Maria Moreno Luzia

E-mail: deboramaria_moreno@yahoo.com.br Curriculum Lattes: http://lattes.cnpq.br/4668535194933989

Doutorado em Engenharia e Ciência de Alimentos pela Universidade Estadual Paulista "Júlio de Mesquita Filho" e graduação em Nutrição pela Universidade do Sagrado Coração. Tem experiência na área de Ciência e Tecnologia de Alimentos, com ênfase na caracterização de resíduos de frutos para obtenção de antioxidantes naturais e compostos bioativos, atuando principalmente nos seguintes temas: frutos brasileiros não convencionais, frutos do cerrado, compostos bioativos (fitosteróis, tocoferóis, ácidos graxos, compostos fenólicos e carotenoides) e atividade antioxidante.

Nome: Neuza Jorge

E-mail: neuza.jorge@unesp.br

Curriculum Lattes: http://lattes.cnpq.br/6605948620230104

Pós-Doutorado na área de Ciência de Alimentos no Departamento de Ciencia de Alimentos y Tecnología Química, Facultad de Ciencias Químicas y Farmacéuticas, Universidad de Chile, Santiago e na área de Bromatologia na Faculdade de Farmácia, Universidade do Porto. Doutorado Sanduiche em Engenharia de Alimentos pelo Instituto de La Grasa - Consejo Superior de Investigaciones Científicas/Universidade Estadual de Campinas, mestrado em Engenharia Agrícola e graduação em Engenharia de Alimentos pela Universidade Federal de Viçosa. Atualmente é professora Associada no Departamento de Engenharia e Tecnologia de Alimentos do Instituto de Biociências, Letras e Ciências Exatas da Universidade Estadual Paulista "Júlio de Mesquita Filho". Atua na área de Ciência e Tecnologia de Alimentos, com ênfase em Matérias Graxas Alimentícias, principalmente nos seguintes temas: frituras, alterações físico-químicas de lipídios, antioxidantes naturais e compostos bioativos em óleos e gorduras. 